\title{
Using spline-extrapolation in the research of self-similar traffic characteristics
}

\author{
Irina Strelkovskaya ${ }^{*}$, Irina Solovskaya ${ }^{* *}$
}

\begin{abstract}
The problem of predicting self-similar traffic is considered, the solution of which modeling of self-similar traffic was performed using the Simulink software package in MATLAB environment. For the simulation, the queuing system WB/M/1/K with Weibull distribution was used. The use of the spline-extrapolation method made it possible to predict self-similar traffic outside the considered period of time on which packet data transmission is considered. Extrapolation of traffic for short-term and long-term forecasts is considered. Comparison of the results of the prediction of self-similar traffic using various spline functions has shown that the accuracy of the forecast can be improved through the use of cubic splines. A method is proposed for estimating the error of traffic prediction for each variant of traffic forecasting using linear, cubic splines. The results of the research will allow you to perform effective traffic management with the support of quality characteristics, by providing the required parameters of network hardware and software in order to avoid overloads in the network.
\end{abstract}

K e y w or d s: self-similar traffic; quality of service; predicting; extrapolation; spline functions; the error of recovery

\section{Introduction}

The rapid development of mobile communication technologies in the direction of $4 \mathrm{G} / 5 \mathrm{G}$ generation networks is connected, first of all, with the development of a wide range of high-speed services provided to users. The most popular today are video services (YouTube, Video Surviliance, Smart-TV, HD-video, 3D-video) and M2M inter-machine interaction services (IoT-cameras, SmartM2M objects, IoT-telematra) Internet of things (IoT). The introduction of multimedia services and interactive applications requires the operator to significantly increase the data transfer rate and ensure the required QoS (quality of service) characteristics. In this case, IoT services are characterized by high-speed video and data traffic $[1,2]$.

In such networks using packet switching, it is required to solve the problem of supporting QoS characteristics: determining the delay time of packets, the probability of their loss, and supporting the required bandwidth. The main difficulty in solving this problem is the self-similar nature of the traffic in question, which manifests itself as a long-term relationship between traffic values at different time periods. Significant and frequent bursts of intensity look statistically similar at different time scales $[3,4]$.

This property allows, based on the accumulated statistical data or based on the results of traffic modeling, to predict its state in the future. In practice, the appearance of a significant amount of long-term pulsations of traffic intensities at arbitrary times often leads to a sharp increase in packet delay time, which causes network nodes and buffer devices to overload and, accordingly, significantly affect the quality of traffic servicing.

Predicting self-similar traffic will allow for possible peak loads in the network and perform efficient traffic management, thus ensuring the required QoS characteristics. Taking into account the above facts, it is possible to note the urgency of solving the problem of predicting self-similar traffic. A number of works by the authors are devoted to the problem of predicting self-similar traffic [5-12]. The proposed models and methods of forecasting are not universal, so autoregression models [5] are used only for processes with poorly expressed selfsimilarity, and models ARIMA and FARIMA [6-8] are recommended only for short-term forecasts. As an alternative method, a method of neural network prediction was proposed in $[9,10]$, allows solving a number of practical forecasting problems, such as dynamic bandwidth redistribution in order to make optimal use of available network resources and maintain QoS characteristics. However, it is important to note that the use of the neural network method implies the need for network training, this is a complex and time-consuming process, and even a "trained" network is not always clearly predictable due to heuristic approaches to its construction. The accuracy of the forecast in such a network depends on the number of options for its training, and the implementation has high requirements for computing capabilities. In [11], the author of the comparison results of network traffic prediction using linear regression models and neural networks shown that in most cases the use of complicated and labor-intensive techniques of neural networks is im-

*A.S. Popov Odessa National Academy of Telecommunications, Department of Higher Mathematics, **Department of Switching Systems, Odessa, Ukraine, Kuznechnaya str., i.strelkovskaya@onat.edu.ua, i.solovskaya@onat.edu.ua 


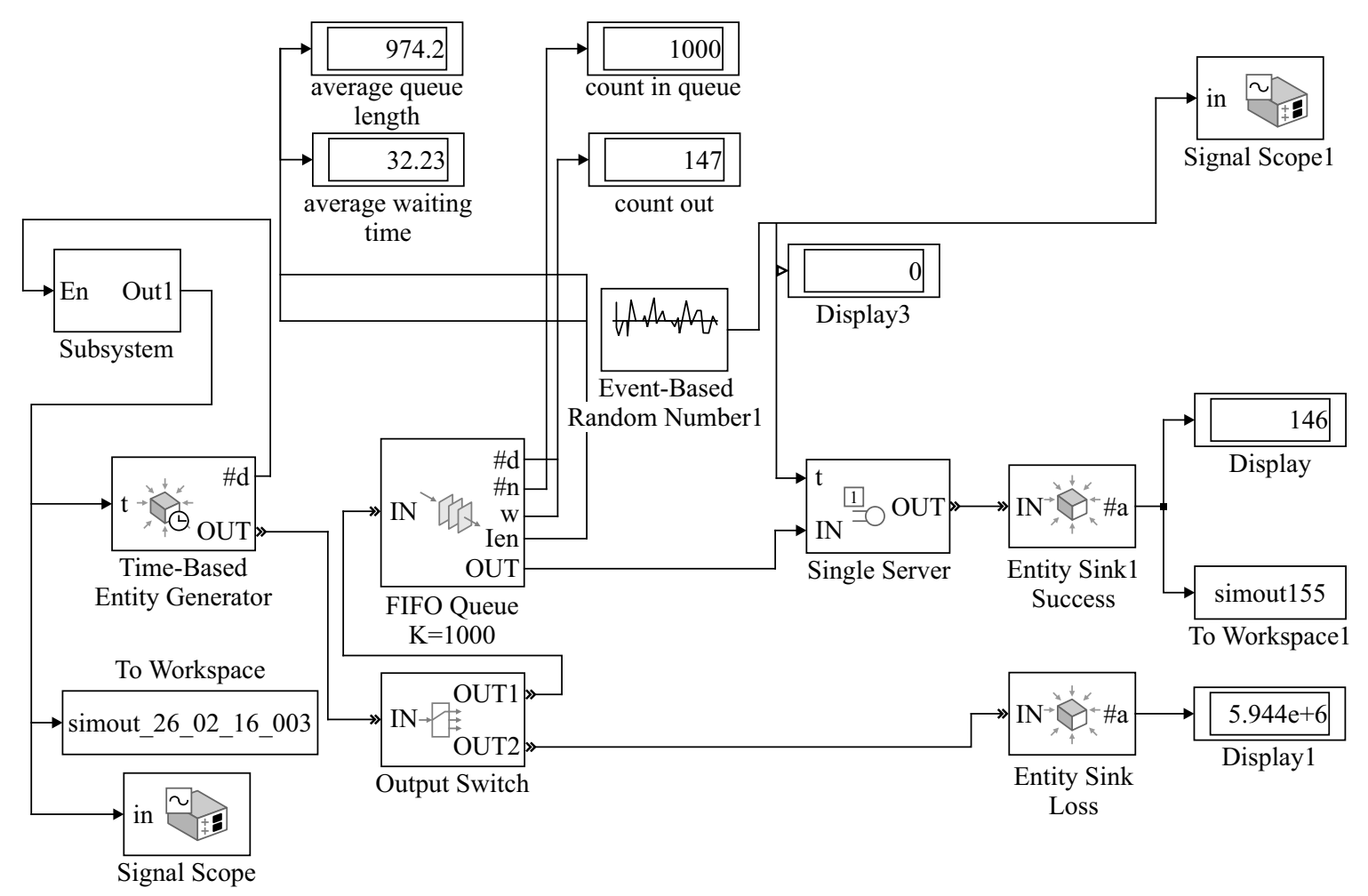

Fig. 1. Simulation of $\mathrm{CMO}$ of type $\mathrm{WB} / \mathrm{M} / 1 / \mathrm{K}$ in simulink package in MATLAB environment

practical. There is no single method for predicting selfsimilar traffic.

Therefore, the development of effective methods for predicting self-similar traffic is only an element in the development of the theory of telecommunication systems, which will allow us to move from purely technological solutions based on engineering intuition to solutions based on systems theory. All this allows us to affirm the significant interest of many scientists to the issue under consideration.

In this regard, the subject of this work is topical and its goal is to solve the problem of predicting self-similar traffic and choosing an approximating device, with which the traffic forecast will be most accurate. This will allow taking timely measures to prevent overloading of network nodes and buffer devices and the corresponding effects of these changes on the quality of service.

\section{Modeling of self-similar traffic using the Simulink package in MATLAB environment}

Let us perform modeling of self-similar traffic for queuing system (QS) of $\mathrm{WB} / \mathrm{M} / 1 / \mathrm{K}$ type that serves the requests stream, which intervals are described by arbitrary distribution WB, the time of service has exponential distribution M, QS has 1 line and length of requests queue is $K$. To develop a simulation model of self-similar traffic, we use the Simulink software package in the Matlab environment, which allows using SimEvents blockset [13] to create different QS with traffic generating sources with different distribution laws and service disciplines, as well as queue maintenance methods, including priorities.

Let us consider the Weibull distribution, given by the differential distribution function [14-17]

$$
f(x)= \begin{cases}\alpha \beta^{\alpha-1} e^{-\beta x^{\alpha}}, & x \geq 0, \\ 0, & x \leq 0\end{cases}
$$

where $\alpha$ is a parameter of Weibull distribution curve form, $0<\alpha<1$, which is determined according to [14-16] by expression $\alpha=2-2 H$, with $H$ being the Hurst parameter, $0.5 \leq H \leq 1$, and $\beta=[\lambda \Gamma(1+1 / \alpha)]^{\alpha}$ the Weibull distribution parameter with $\beta>0, \lambda$ is the intensity of requests arrivals for QS servicing, $\Gamma(k)$ is the Euler gamma function

$$
\Gamma(k)=\int_{0}^{\infty} t^{k-1} e^{-t} \mathrm{~d} t .
$$

The integral function of Weibull distribution is [14]

$$
F(x)=1-e^{-\beta x^{\alpha}} .
$$

To generate a sample of random variables with the Weibull distribution, let us find the inverse function of the integral distribution function (2), solving the latter for the unknown $x$ we obtain

$$
x=\left[-\frac{1}{\beta} \ln (1-y)\right]^{\frac{1}{\alpha}} .
$$




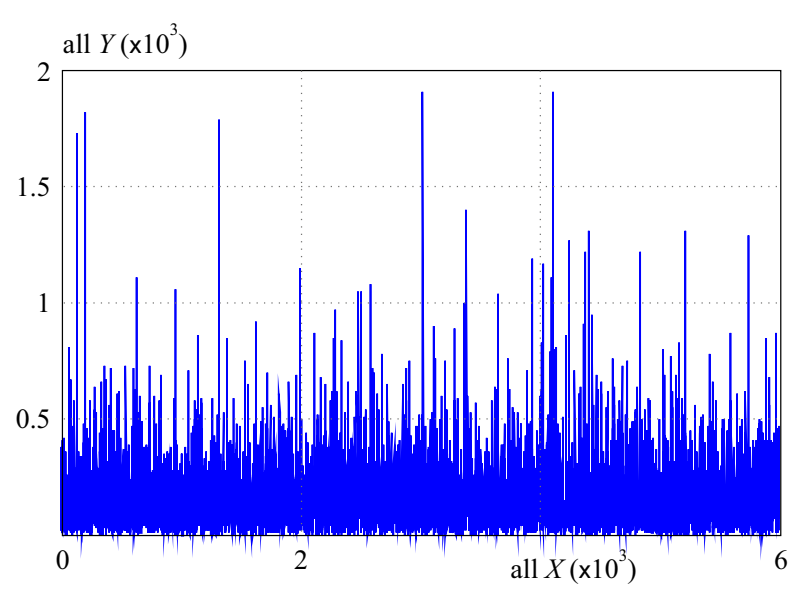

Fig. 2. Results of self-similar traffic modelling for QS type $\mathrm{WB} / \mathrm{M} / 1 / \mathrm{K}$

Thus, the inverse function, which is determined finite only on the interval $(0,1)$, is

$$
F^{-1}(y)=\left[-\frac{1}{\beta} \ln (1-y)\right]^{\frac{1}{\alpha}}
$$

Having a sequence of uniformly distributed random numbers, we can calculate the values of random numbers with the Weibull distribution and the mean value

$$
\mu=\beta^{\frac{1}{\lambda}} \Gamma\left(1+\frac{1}{\lambda}\right)
$$

using the following algorithm

$$
X_{i}=\left[-\frac{1}{\beta} \ln \left(1-U_{i}\right)\right]^{\frac{1}{\alpha}}
$$

where $U_{i}$ denotes random values uniformly distributed within the interval $(0,1)$.

As the initial data of $\mathrm{QS} \mathrm{WB} / \mathrm{M} / 1 / \mathrm{K}$ operation, we use its following characteristics: $\lambda=250 \mathrm{pack} / \mathrm{s}$, is intensity of packets arrivals for servicing in QS, $\mu=150 \mathrm{~s}$ is packets servicing durability, $K=1000$ packets is the length of packets queue. The QS model of WB/M/1/K type, developed in the Simulink package (the MATLAB environment) is shown in Fig. 1.

Figure 2 shows the results of simulating self-similar traffic for QS of type $\mathrm{WB} / \mathrm{M} / 1 / \mathrm{K}$, where $N$ is the number of applications; it is a time of receipt of applications.

The model consists of a single source that generates packets using the developed Weibull Distribution Generator, which generates a sequence of random numbers (5) with the specified Weibull distribution parameters $(\alpha \approx 0.4, \beta \approx 13.46)$ and intensity $\lambda=250 \mathrm{pack} / \mathrm{s}$, Hurst parameter $H=0.8$.

All packages are served by the Output Switch information distribution system with a service time of $\mu=150 \mathrm{~s}$, exponentially distributed in the Single Server. The discipline of service in the QS, with waiting, the queue length of packets is $K=1000$, and the packet maintenance is performed in the queue according to the FIFO principle in the Queue "first come, first come out". During maintenance, packets are registered in Entity Sink Success, and in case of losses arising in the event of a buffer device overflow in Entity Sink Loss.

For demonstration of simulation results, windows are used that record the results of servicing traffic in a QS of the $\mathrm{WB} / \mathrm{M} / 1 /$ Ktype in real time: Average waiting time, Average queue length, Count in queue is queue length packets and Count out is number is lost of packets [12].

According to Fig. 2, for self-similar traffic in the interval $[0,6000]$ ms scale invariance, the presence of "bursts" of packages and a long-term relationship between the moments of their arrival are observed.

\section{Prediction of the self-similar traffic}

To predict the self-similar traffic, we use the extrapolation method. The research task is as follows:

1) predict the traffic beyond the considered time period, where the transmission of packet data is considered;

2) choose an approximating device, with the help of which it is possible to perform a more accurate prediction of self-similar traffic; and

3) determine the error in restoring self-traffic outside the gap.

Earlier, problem solving extrapolation of random processes was based on the use of Lagrange interpolation polynomials, Chebyshev polynomials, etc. When predicting self-similar traffic, we use the extrapolation method based on the spline-function, since [14]:

1) splines are more resistant to local perturbations, that is, the behavior of the spline in the neighborhood of the point does not affect the spline behavior as a whole, as, for example, this occurs in polynomial interpolation; and

2) good convergence of spline-interpolation as opposed to polynomial interpolation. In particular, splineinterpolation is an indisputable priority for functions with irregular smoothness properties (an example of which self-similar traffic serves).

The task of predicting self-similar traffic is solved by the "spline-extrapolation" method, that is, the recovery of self-similar traffic outside the considered time interval based on spline-functions (for example, linear, cubic, etc). Considering that self-similar traffic is characterized by the presence of "bursts" packets, it is possible to use extrapolation based on wavelet-functions to improve the accuracy of prediction.

Let us dwell on the method of spline-extrapolation. With the term spline-extrapolation we mean an extrapolation based on the use of spline functions. Consider self-similar traffic on the interval $[a, b]$. Let a partition $\Delta: a=x_{0}<x_{1}<\cdots<x_{N}=b$ be given. The firstdegree spline $S_{1}(x)$ on the grid $\Delta$ is a continuous piecewise linear function. Let the grid points be given values of 


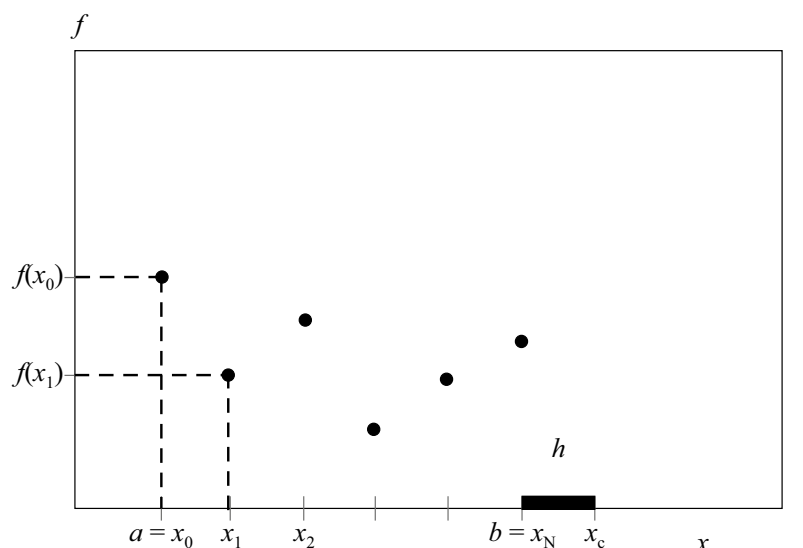

Fig. 3. The extrapolation of self-similar traffic on the interval $[a, b]$ under condition $f\left(x_{c}\right)=f\left(x_{1}\right)$

self-similar traffic $f_{i}=f\left(x_{i}\right)$, that describe the function $f(x)$, defined on interval $[a, b]$. The interpolation spline is defined by the following conditions

$$
S_{1}\left(x_{i}\right)=f_{i}, \quad i-1, \ldots, N \text {. }
$$

Geometrically, it is a polygonal line passing through the points $\left(x_{i}, y_{i}\right)$, where $y_{i}=f\left(x_{i}\right)$. Let $h_{i}=x_{i+1}-x_{i}$. Then, according to [18], for $x \in\left[x_{i}, x_{i+1}\right], i=0, \ldots, N-1$, the linear spline will be

$$
S_{1}(x)=f_{i} \frac{x_{i+1}-x}{h_{i}}+f_{i+1} \frac{x-x_{i}}{h_{i}}
$$

or

$$
S_{1}(x)=f_{i}+\frac{x-x_{i}}{h_{i}}\left(f_{i+1}-f_{i}\right) .
$$

We will also consider a cubic interpolation spline $S_{3}(x)$, constructed similarly to a linear spline, with the only difference being that this is a cubic function on each interval $\left[x_{i}, x_{i+1}\right], i=1, \ldots, N-1$.

According to [18], for $x \in\left[x_{i}, x_{i+1}\right], i=1, \ldots, N-1$ the cubic spline has the form:

$$
\begin{aligned}
S_{3}(x)=f_{i}(1-t)^{2} & (1+2 t)+f_{i+1} t^{2}(3-2 t)+ \\
& m_{i} h_{i} t(1-t)^{2}-m_{i+1} h_{i} t^{2}(1-t),
\end{aligned}
$$

where $t=\frac{x-x_{i}}{h_{i}}, S_{3}\left(x_{i}\right)=f_{i}, S_{3}\left(x_{i+1}\right)=f_{i+1}, m_{i}=$ $S^{\prime}\left(f ; x_{i}\right)$, or

$$
\begin{aligned}
S_{3}(x)=f_{i}(1-t)+f_{i+1} t & - \\
& \frac{h_{i}^{2}}{6} t(1-t)\left[(2-t) M_{i}+(1+t) M_{i+1}\right],
\end{aligned}
$$

where $S^{\prime \prime}\left(x_{i}\right)=M_{i}, S^{\prime \prime}\left(x_{i+1}\right)=M_{i+1}$.

The boundary conditions are used to determine the cubic spline of the form (9) on the interval $[a, b][18]$

$$
\left.\left.S_{(}^{\prime} f ; a\right)=f^{\prime}(a), \quad S_{(}^{\prime} f ; b\right)=f^{\prime}(b) .
$$

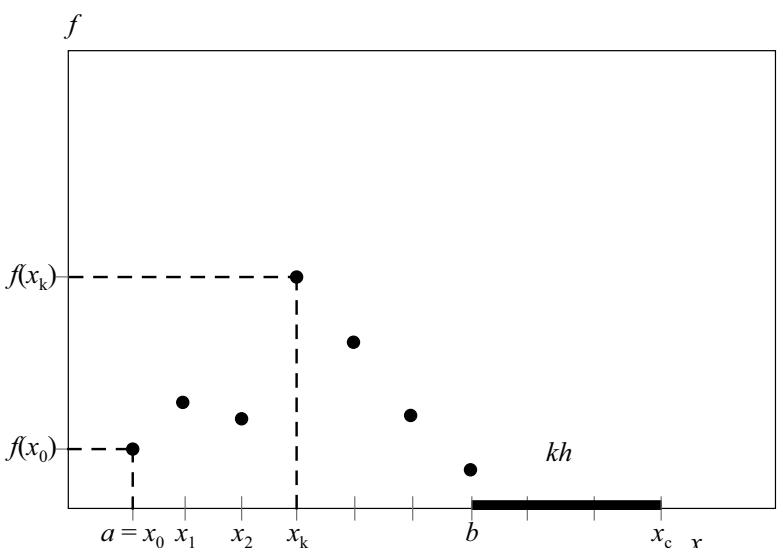

Fig. 4. The extrapolation of self-similar traffic on the interval $[a, b]$ under condition $f\left(x_{k h}\right)=f\left(x_{c}\right)$

For determining a cubic spline type (10) using the boundary conditions of the form [18]

$$
S^{\prime \prime}(f ; a)=f^{\prime \prime}(a), \quad S^{\prime \prime}(f ; b)=f^{\prime \prime}(b)
$$

We consider a uniform partition of the interval $[a, b]$, ie

$$
h_{i}=\frac{b-a}{N}, \quad i=0,1, \ldots, N-1 .
$$

It is necessary to restore the self-similar traffic outside the interval $[a, b]$, namely, the right of the point $b$. For definiteness, let this be a point $x_{c}>x_{N}=b, x_{c}-b=h$, where $h$ is a partition step of interval $[a, b]$. Then we construct spline-function (linear or cubic) on the interval $\left[b, x_{c}\right]$.

Let us consider two variants. In the first case, we assume that $f\left(x_{c}\right)=f\left(x_{1}\right)$ and construct the spline in interval $\left[b, x_{c}\right]$, Fig. 3 .

In the second case, Fig. 4 , we set $f\left(x_{k}\right)=f\left(x_{c}\right)$, where $x_{k}=x_{0}+k h$, where $k$ is any natural number. If $k h x_{F} x_{c}-b$, then for $f\left(x_{c}\right)$ we take the value of the function $f(x)$ which are closest to the point $x_{k}$.

According to [18], it is not difficult to find the error of restoring self-similar traffic on the interval $\left[b, x_{c}\right]$, using the following theorems.

Theorem 1 [18]. If a spline of the first degree $S_{1}(x)$ interpolates a continuous function $f(x)$ on the grid $\Delta$, then its estimation of the error is

$$
\begin{gathered}
\left|S_{1}(x)-f(x)\right| \leq \omega(f), \\
\omega(f)=\max _{0 \leq i \leq N-1} \omega_{i}(f), \\
\text { where } \omega_{i}(f)=\max _{x^{\prime}, x^{\prime \prime} \in\left[x_{i}, x_{i+1}\right]} \mid f\left(x^{\prime \prime}-f\left(x^{\prime}\right) \mid .\right.
\end{gathered}
$$

TheOREM 2 [18]. If the cubic spline $S_{3}(x)$ interpolates a continuous function $f(x)$ on the net $\Delta$ and satisfies the boundary conditions (11) or (12), then

$$
\left\|S_{3}(x)-f(x)\right\|_{C} \leq\left(1+\frac{3}{4}\right) \omega(f),
$$




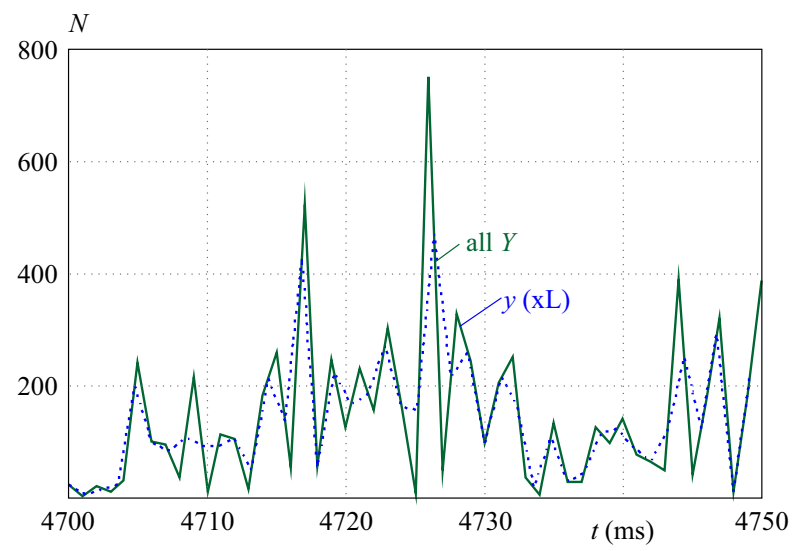

Fig. 5. Results of extrapolation of self-similar traffic on the interval $[4700,4750]$ ms using linear spline

where $\rho=\frac{\max _{i} h_{i}}{\min _{i} h_{i}},\|f(x)\|_{C}=\max _{x \in[a, b]}|f(x)|$, $C=[a, b]$ is the space of all continuous functions defined on $[a, b]$.

\section{Solution of the problem of prediction self-similar traffic}

Consider the spline-extrapolation using the results of simulating self-similar traffic with various linear and cubic splines. Consider the extrapolation of traffic shown in Fig. 2, on the interval $[4700,4750] \mathrm{ms}$ and compare with the obtained results of the simulation of self-similar traffic.

The first variant of spline-extrapolation, Fig. 3, allows to perform a short-term traffic prediction. In this case, the prediction of traffic characteristics occurs in real time, which allows the control and dynamic redistribution of network resources depending on the behavior of traffic, for example, frequent "bursts" of intensity.

We use the linear spline $S_{1}(x),(6)-(8)$ for the first version of the spline-extrapolation of the simulated selfsimilar traffic on the $[4700,4750] \mathrm{ms}$ segment, Fig. 5.

It is not difficult to see, Fig. 5, that the use of the linear spline functions has a significant error, which most often appears on segments where traffic intensity graphs have periodic "bursts", the extrapolation error of self-similar traffic on the interval $[4700,4750] \mathrm{ms}$ are shown in Tab. 1.

Consider the first variant of spline-extrapolation (short term prediction) for simulated self-similar traffic in the $[4700,4750] \mathrm{ms}$ segment using the cubic spline $S_{3}(x),(9)$, (10), see Fig. 6.

According to the results of the extrapolation of selfsimilar traffic on segment $[4700,4750]$ ms using the cubic spline function shown in Fig. 6, the recovery errors are obtained on the segments of the graph, where the traffic intensity has "spikes" at peak points, the error shown in Table 1. In general, the use of cubic splines allows you to perform a short-term forecast of traffic parameters and get the predicted "route" of self-similar traffic.

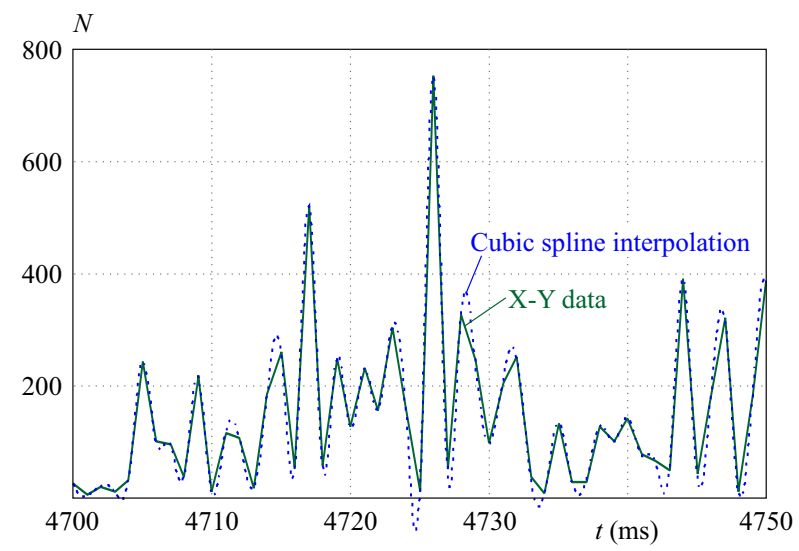

Fig. 6. Results of extrapolation of self-similar traffic on the interval $[4700,4750] \mathrm{ms}$ using cubic spline

The error in recovering self-similar traffic based on a linear and cubic spline (13)-(15) will be evaluated according to Theorems 1 and 2 . The calculation results are shown in Tab. 1.

In general, the proposed extrapolation method based on spline functions, according to the authors, has a number of advantages in comparison with the known methods. It is quite simple to implement, has a small error, and can also be used to control traffic in real time.

Table 1. The error of recovery of self-similar traffic based on a linear and cubic spline

\begin{tabular}{cccc}
\hline Interval & Time & \multicolumn{2}{c}{ Spline } \\
& $(\mathrm{ms})$ & linear & cubic \\
\hline$[\mathrm{x} 10, \mathrm{x} 11]$ & {$[4710,4711]$} & 6.12 & 1.02 \\
{$[\mathrm{x} 11, \mathrm{x} 12]$} & {$[4711,4712]$} & 10.81 & 0.05 \\
{$[\mathrm{x} 12, \mathrm{x} 13]$} & {$[4712,4713]$} & 16.98 & 2.52 \\
{$[\mathrm{x} 13, \mathrm{x} 14]$} & {$[4713,4714]$} & 14.20 & 1.79 \\
{$[\mathrm{x} 14, \mathrm{x} 15]$} & {$[4714,4715]$} & 21.38 & 7.11 \\
$\vdots$ & $\vdots$ & $\vdots$ & $\vdots$ \\
{$[\mathrm{x} 20, \mathrm{x} 21]$} & {$[4720,4721]$} & 13.75 & 0.24 \\
{$[\mathrm{x} 21, \mathrm{x} 22]$} & {$[4721,4722]$} & 18.79 & 0.93 \\
{$[\mathrm{x} 22, \mathrm{x} 23]$} & {$[4722,4723]$} & 9.31 & 0.75 \\
\hline
\end{tabular}

\section{Discussion}

The long-term traffic forecast allows results that are based on a large amount of data to be obtained and allows the choice of the size of the buffer devices of network nodes and providing for the length of the packet queue in these devices when designing a network.

For the study, simulated self-similar traffic was used for the QS WB/M/1/K using the Simulink package in Matlab with the Hurst coefficient $H=0.8$ (Fig. 1). The high value of the Hurst parameter is accompanied by high 
traffic burst and the presence of significant and frequent bursts of traffic intensity, which is observed in Fig. 2 .

From Fig. 2 it can be seen that for the obtained selfsimilar traffic in $[0,6000] \mathrm{ms}$ segment, there is large-scale traffic invariance, the presence of packet "bursts" and a long-term relationship between the moments of their arrival. It is the presence of a significant number of frequent "bursts" of traffic intensity that leads to a sharp increase in packet delay time, which causes overloading of network nodes and buffer devices and, accordingly, has a significant impact on the quality of service of traffic.

Using linear spline functions, as can be seen from the Tab. 1, has a significant error in some segments of extrapolation. At the same time, it is not difficult to see in Fig. 5, that the error is noted on the segments with significant periodic "bursts" of traffic. From this it follows that the use of linear spline functions does not allow extrapolating bursts of traffic intensity. For example, at the interval $[4716,4717] \mathrm{ms}$ and $[4726,4727] \mathrm{ms}$, the linear spline restores the intensity value of traffic only by 20-25\%. These results were obtained for a given segment of traffic prediction $[4700,4750] \mathrm{ms}$, with a small partitioning step of $1 \mathrm{~ms}$. Increasing the partitioning step will inevitably lead to an increase in the recovery error values. When building a spline-extrapolation, it is important to choose the size of the prediction segment. In this case, the traffic forecast is short-term in the $[4700,4750] \mathrm{ms}$ interval.

Therefore, under the given conditions of traffic forecasting, the use of linear spline functions seems to be inappropriate to the authors, since it does not allow ensuring the required accuracy of the short-term forecast. In practice, short-term forecasts are used in traffic management and routing, and decisions are made in real time, which leads to a decrease in the quality of QoS, especially for high-speed data traffic and high-definition video.

During the experiment, self-similar traffic with the Hurst coefficient $H=0.8$ was used in the work. It is possible that for traffic with a lower Hurst coefficient and other self-similar traffic, the use of spline-extrapolations based on linear splines will provide the required forecast accuracy.

The use of cubic spline functions for extrapolation of self-similar traffic, characterized by frequent intensity "bursts" compared with the use of linear spline functions, makes it possible to achieve a decrease in error values throughout the extrapolation interval $[4700,4750] \mathrm{ms}$. It is not difficult to see in Fig. 6, that the errors are obtained on segments of the "bursts" of traffic intensity at peak points. For example, this is observed at $[4716,4717] \mathrm{ms}$ and $[4726,4727] \mathrm{ms}$, with a sharp increase and decrease in traffic intensity. It is possible to increase the accuracy of prediction of traffic characteristics using waveletextrapolation.

In general, the proposed extrapolation method based on spline functions, according to the authors, has a number of advantages in comparison with the known methods.
It is quite simple to implement, has a small error, and can also be used to control traffic in real time.

The practical significance of the results obtained is that the obtained results of predicting self-similar traffic will provide for the required amount of buffer devices, thereby avoiding overloads in the network and exceeding the standard values of QoS characteristics.

Based on the results of traffic forecasting taking into account the maximum workloads of network nodes, practical recommendations can be given on traffic redistribution over IP networks, for example, the operation of TCP/IP protocol. Reduce the delay time compared with the TCP protocol, allows the protocol without the guaranteed delivery of UDP. However, it is rather difficult to provide the required QoS quality characteristics using only the UDP/TCP transport protocol, since the reasons causing the delays are mostly at the network level. Using the proposed spline-extrapolation method will allow you to perform prediction of traffic characteristics, balance the loading of network objects and improve the efficiency of network equipment use.

\section{Conclusions}

- The solution of the problem of predicting the selfsimilar traffic received using the Simulink software package in the Matlab environment is considered.

- The proposed method of extrapolation based on spline functions has a number of advantages in comparison with known methods. First of all it is easy enough to implement, requires low resource consumption for the accuracy of the forecast, which can be enhanced through the use of quadratic or cubic splineinterpolation functions.

- Using the spline-extrapolation prediction results obtained self-similar traffic, which will provide the required amount of buffer devices, thus avoiding network congestion and exceeding regulatory QoS characteristics values.

- Using the spline-extrapolation, the results of prediction of self-similar traffic were obtained, which will allow to foresee the required amount of buffer devices, thereby avoiding congestion in the network and exceeding the standard values of QoS characteristics.

- Taking into account that the self-similar traffic is characterized by the presence of "bursts" and the longterm dependence between the time of receipt of applications in this study with given initial data, it is possible to use extrapolation based on wavelet functions, the so-called "wavelet-extrapolation" method to increase the prediction accuracy. 


\section{REFERENCES}

[1] 3GPP Study on Scenarios Requirements for Next Generation Access Technologies, ETSI TR 38.913, V14.3.0, 2017.

[2] D. Evans, The Internet of Things, How the Next Evolution of the Internet Is Changing Everything, White Paper: Cisco, 2011.

[3] V. V. Krylov and S. S. Samohvalova, Theory of telegraphic its applications, BXV-Petersburg, 2005.

[4] O. I. Sheluhin, A. V. Osin, and S. M. Smolskii, Self-Similarity Fractals, Telecommunication Applications, Phismatlit, 2008.

[5] F. C. Pereira, C. Antoniou, J. A. Fargas, and M. Ben-Akiva, "A Metamodel for Estimating Error Bounds in Real-Time Traffic Prediction Systems", IEEE Transportation on Intelligent Transportation Systems, vol. 15, no. 3, pp. 1310-1322, 2014, DOI: 10.1109/TITS.2014.2300103, 2014.

[6] C. Y. Li, Z. Han, and Z. Sun, "A novel Self-Similar Traffic Prediction Method Based on Wavelet Transform for Satellite Internet", EAI Endorsed Transactions on Ambient System, vol. 4, no. 14, pp. 1-7, DOI: 10.4108/eai.28-8-2017.153306, 2017.

[7] H. H. Theyazn Aldhyani and J. Manish, "An Integrated Model for Prediction of Loading Packets in Network Traffic", Proceeding Second International Conference on Information Communication Technology for Competitive Strategies, 4-5 March 2016, pp. 1-8, DOI: 10.1145/2905055.2905236, 2016.

[8] V. Kumar and L. Vanajakshi, "Short-term traffic flow prediction using seasonal ARIMA model with limited input data", European Transport Research Review, Springer, vol. 7-21, pp. 9-21, 2015, DOI: $10.1007 / \mathrm{s} 12544-015-0170-8$.

[9] P. Sarigiannidis, K. Aproikidis, M. Louta, and P. Angelidis, "Predicting Multimedia Traffic in Wireless Networks: A Performance Evaluation of Cognitive Techniques", Proceeding 5th International Conference on Information, Intelligence, Systems Applications (IISA-2014), 7-9 July 2014, pp. 341-346, 2014, DOI: $10.1109 /$ IISA.2014.6878802.

[10] M. Oravec, M. Petras, and P. Pilka, "Video Traffic Prediction Using Neural Networks", Acta Polytechnica Hungarica, vol. 5, no. 4, pp. 59-78, 2008

[11] I. Klevecka, "Forecasting Network Traffic: A Comparison of Neural Networks Linear Models Reliability Statistics in Transportation Communication", Proceeding 9th International Conference (RSTC-2009), 21-24 October 2009, pp. 31-36, 2009.

[12] E. Grab and S. Sharkovsky, "Real-Time Estimation of Traffic Self-Similarity Parameter in Simulink with Wavelet Transform", Elektronika ir elektrotechnika, vol. 19, pp. 88-91, 2008.

[13] https://www.mathworks.com.

[14] I. V. Strelkovskaya, I. N. Solovskaya, N. V. Severin, and S. A. Paskalenko, "Spline Approximation based Restoration for Self-Similar Traffic", Eastern-European Journal of Enterprise Technologies, vol. $3 / 4$, no. 87 , pp. 45-50, 2017, DOI: 10.15587/1729-4061.2017.102999.
[15] I. Strelkovskaya and I. Solovskaya, "Approximation of Self-Similar Traffic by Spline-Functions", Proceedings of the XIII-th International Conference Modern Problems of Radio Engineering, Telecommunications Computer Science: (TSET'2016), 23-26 February, 2016, pp. 132-135, 2017, DOI: 10.1109/tcset.2016.7451991.

[16] I. V. Strelkovskaya, T. I. Grygoryeva, and I. N. Solovskaya, "Self-Similar Traffic in G/M/1 queue defined by the Weibull Distribution", Radioelectronics Communications Systems, vol, 61, no. 3, pp. 173-180, 2018, DOI: 10.20535/S0021347018030056.

[17] I. Strelkovskaya, I. Solovskaya, and A. Makoganiuk, "Optimization of QoS chracteristics of self-similar traffic", Proceeding of the 4th International Scientific-Practical Conference: Problems of Infocommunications Science Technology (PICS\&T 2017), 10-13 October 2017, pp. 497-500, 2017, DOI: 10.1109/INFOCOMMST.2017.8246447.

[18] Yu. S. Zavyalov B. I. vasov and V. L. Miroshnichenko, Methods of Spline Functions, Science, 1980.

Received 6 June 2019

Strelkovskaya Irina, received her PhD degree in Mathematical Analysis from the Donetsk State University, DSc degree in Telecommunication Systems and Networks from the A.S. Popov Odessa National Academy of Telecommunications (2010) and academic rank of Professor (2011). Since 2000 to the present time she is the head of the Educational and Research Institute of Infocommunications and Software Engineering in A.S. Popov Odessa National Academy of Telecommunication. She is the author of more than 253 scientific and educational works, among which: 178 scientific articles and conference abstracts, 4 patents for invention, 10 textbooks, 2 monographs, 59 educational and methodical manuals. Her current research interests includes the study of analytic splines in the areas with quasiconformal boundary, application of splineand wavelet- apparatus in telecommunication theory, tensor analysis to solve telecommunication problems.

Solovskaya Irina, received her PhD degree in Telecommunication Systems and Networks from the A. S. Popov Odessa National Academy of Telecommunications, Associate Professor in the Department of Switching Systems. She is the author of more than 70 scientific and educational works, including 60 scientific articles and conference abstracts, 10 educational and methodical manuals, 1 monograph. Her research interest includes the application of spline- apparatus and tensor method in solving telecommunications problems. 